\title{
Characterization of amorphous ribbon by means vibrating sample magnetometry as an interesting tool to investigate a possible detector of vector field
}

\author{
Arturo Mendoza Castrejón, ", Herlinda Montiel Sánchez², Guillermo Alvarez Lucio 3 , \\ Damasio Morales Cruz ${ }^{1}$ \\ ${ }^{1}$ School of Mechanical and Electrical Engineering -IPN, 07738, U. P. Adolfo López Mateos, D. F., México \\ ${ }^{2}$ Technosciences Department, Center for Applied Science and Technology Development -UNAM, 04510, C.U., D. F., México \\ ${ }^{3}$ School of Physics and Mathematics -IPN, 07738, U. P. Adolfo López Mateos, D. F., México
}

\section{Email address: \\ zaratustra_also@hotmail.com (A. M. Castrejón)}

\section{To cite this article:}

Arturo Mendoza Castrejón, Herlinda Montiel Sánchez, Guillermo Alvarez Lucio, Damasio Morales Cruz. Characterization of Amorphous Ribbon by Means Vibrating Sample Magnetometry as an Interesting Tool to Investigate a Possible Detector of Vector Field. International Journal of Mechanical Engineering and Applications. Vol. 2, No. 6, 2014, pp. 111-116. doi: 10.11648/j.ijmea.20140206.15

\begin{abstract}
Characterization of amorphous ribbon is made by using Vibrating Sample Magnetometry VSM technique with different geometric arrangements: P10, P190, P20 and P290. The purpose is to determine the evolution of the saturation magnetization $M_{S}$, retentivity $M_{R}$ and magnetic anisotropy $K_{1}$ as a function of annealing time treatment and also as a function of the geometric arrangement. The rate of change of magnetization $\Delta \mathrm{M} / \Delta \mathrm{H}$ is determined for orientation P190 and orientation P290. These values of rate of change for the ribbon with no annealing treatment are: $0.122 \mathrm{emu} / \mathrm{cm}^{3}$ and $0.11 \mathrm{emu} / \mathrm{cm}^{3}$, respectively. The highest values of anisotropy are for orientation P190 and for orientation P290, these values are: $K_{1}=2,365,100 \mathrm{erg} / \mathrm{cm}^{3}$ and $\mathrm{K}_{1}=2,405,520 \mathrm{erg} / \mathrm{cm}^{3}$, respectively. Thus we establish that the amorphous ribbon is a strong candidate for technological applications in the area of the magnetic industry, because they can be designed vector field detectors in three directions: longitudinal, transverse (to the ribbon axis) and normal to ribbon plane.
\end{abstract}

Keywords: Retentivity, Magnetic Anisotropy, Saturation Magnetization

\section{Introduction}

Due to rapid technological progress, we have seen an increasing interest in materials that can respond quickly to excitation fields; in particular we refer to ferromagnetic materials which respond to DC magnetic fields. We can find many techniques for determining the magnetic response of materials, such as magneto-optical method, vibrating-coil magnetometer and magnetic force, between some others. However, in this paper the vibrating-sample magnetometer is used because it minimizes any error source, additionally the technique is simple, inexpensive and most importantly, it allows high accuracy in the measurement of the magnetic moment.

A field in a volume element generates an energy gradient by means a force; this force can be detected by the moving of the charge carrier or by the torque on the magnetic dipoles. Both these effects cause changes in the magnetic structure, which is formed by longitudinal and transverse domains (longitudinal and transverse anisotropy) [1,2]. These effects lead to changes in material properties and these properties are explained because the nanocrystals length $\mathrm{D}$ is smaller than the exchange length $L_{e x}[3-5]$, see (1),

$$
\langle K\rangle=\frac{v_{c r}^{2} D^{6}}{A^{3}} K_{1}^{4}
$$

$\langle K\rangle$ is the average energy density anisotropy, $v_{c r}$ is the volume fraction, $\mathrm{D}$ is the grain size, $A$ is a constant depending on the exchange length $L_{e x} y K_{1}$ is the anisotropy energy. Thus, a very important factor to understand the micro-structural properties is to know the magnetic anisotropy and how to control it. For this purpose we use the Vibrating Sample Magnetometry VSM which was first described by Foner [6] and it is based on the change of flow in a coil when the sample vibrates perpendicular to magnetic field, this vibration causes a change of a scalar potential in the form $\phi_{1} \exp (i \omega t)$, where 
$\phi_{1}=-a(\partial \phi / \partial z)$, with sufficiently small amplitude $a, \phi$ is the scalar potential of a fixed dipole, this technique gives information about the magnetization $M$ [7]. Since this technique is very versatile and highly sensitive, in this work will made a characterization to establish both the magnetic properties and the geometrical conditions for the development of a measurement methodology and thereby propose criteria for the possible development of a vector field sensor.

\section{Experimental Procedure}

The alloy $\mathrm{Fe}_{73.5} \mathrm{~B}_{9} \mathrm{Si}_{13.5} \mathrm{Mo}_{3} \mathrm{Cu}_{1}$ was made at Materials Research Institute, México, using a conventional melt spinning method in a protective argon atmosphere, a casting speed of $40 \mathrm{~m} / \mathrm{s}$ was employed. The resulting ribbon was 3 mm wide and $25 \mu \mathrm{m}$ thick. Vibrating-sample magnetometer (LDJ 9600 model) is used, in sweep magnetic field cycle $\mathrm{H}_{\mathrm{DC}}$ $= \pm 1000$ Oe.

Four configurations were used: 1) P10, the plane of the amorphous ribbon $\mathrm{AR}$ is in the $y z$ plane, the $y$ axis is parallel to the longitudinal axis LA of ribbon ( $y \| \mathrm{LA})$, the $z$ axis is parallel to the transverse axis TA of ribbon $(z \| \mathrm{TA})$; 2) P190, the ribbon plane is in the $x z$ plane, $x\|\mathrm{LA}, z\| \mathrm{TA}$; 3) $\mathrm{P} 20$, the ribbon plane is in the $y z$ plane, $z\|\mathrm{LA}, y\| \mathrm{TA}$; and 4) $\mathrm{P} 290$, the ribbon plane is in the $x z$ plane, $z \|$ LA, $x \|$ TA. The $\mathrm{H}_{\mathrm{DC}}$ is always in the $y$ axis direction $\left(\mathrm{H}_{\mathrm{DC}} \| y\right)$. The furnace annealing was performed up to $400 \mathrm{C}$ in a hydrogen flow atmosphere at different annealing times 10, 4060120 and $180 \mathrm{~min}$. Magnetic properties were measured on sample with $6 \mathrm{~mm}$ long, retentivity $M_{R}$, saturation magnetization $\mathrm{M}_{\mathrm{S}}$, anisotropy energy $\mathrm{K}_{1}$ were determined from hysteresis loop.

\section{Results and Discussions}

In a traditional VSM, when the orientation of the sample to the field is changed, the orientation of the sample relative to the coils is changed. As an important result, the response and the sensitivity of the sample will be different at every geometrical configuration (P10, P190, P20 and P290). This is especially true if the sample is not rotation symmetric, it is because we have a sample with longitudinal y transversal anisotropies. Since the sample has a positive magnetostriction and contains $73.5 \%$ iron, a longitudinal anisotropy due to the spin-orbit interaction is generated. Although the presence of copper is lower, only $1 \%$, this also generates a transverse anisotropy. Even with rotation symmetric samples there will always be some angular variation due to rotation eccentricities We show how depending on the orientation of the amorphous ribbon with respect to an applied external field, it has a characteristic response of the longitudinal and transverse domains, this answer can be see because there are important changes in: the anisotropy energy $K_{1}$, the retentivity $M_{R}$ and the saturation magnetization $\mathrm{M}_{\mathrm{S}}$. High sensitivity of the material relative to field is shown in Fig. 1, the dotted line box represents the region of magnetic field created by the electromagnet poles, the field is always in the direction of the $y$ axis. We can observe two important facts: the first, a clear difference in the hysteresis loops between $\{\mathrm{P} 10, \mathrm{P} 20\}$ and $\{\mathrm{P} 190, \mathrm{P} 290\}$, this is because in $\{\mathrm{P} 10, \mathrm{P} 20\}$ the magnetic moment dynamic is in ribbon plane, but not for $\{\mathrm{P} 190, \mathrm{P} 290\}$ where the dynamic of the moments is out of plane; the second, a difference between $\{\mathrm{P} 10, \mathrm{P} 20\}$ and $\{\mathrm{P} 190, \mathrm{P} 290\}$, it is explained in terms of the effect of the field on the transverse and longitudinal anisotropy, respectively. These important facts establish the basic conditions to design of vector field sensors. But let analyze the properties of the amorphous ribbon, which determine the suitability of a material for a given application.

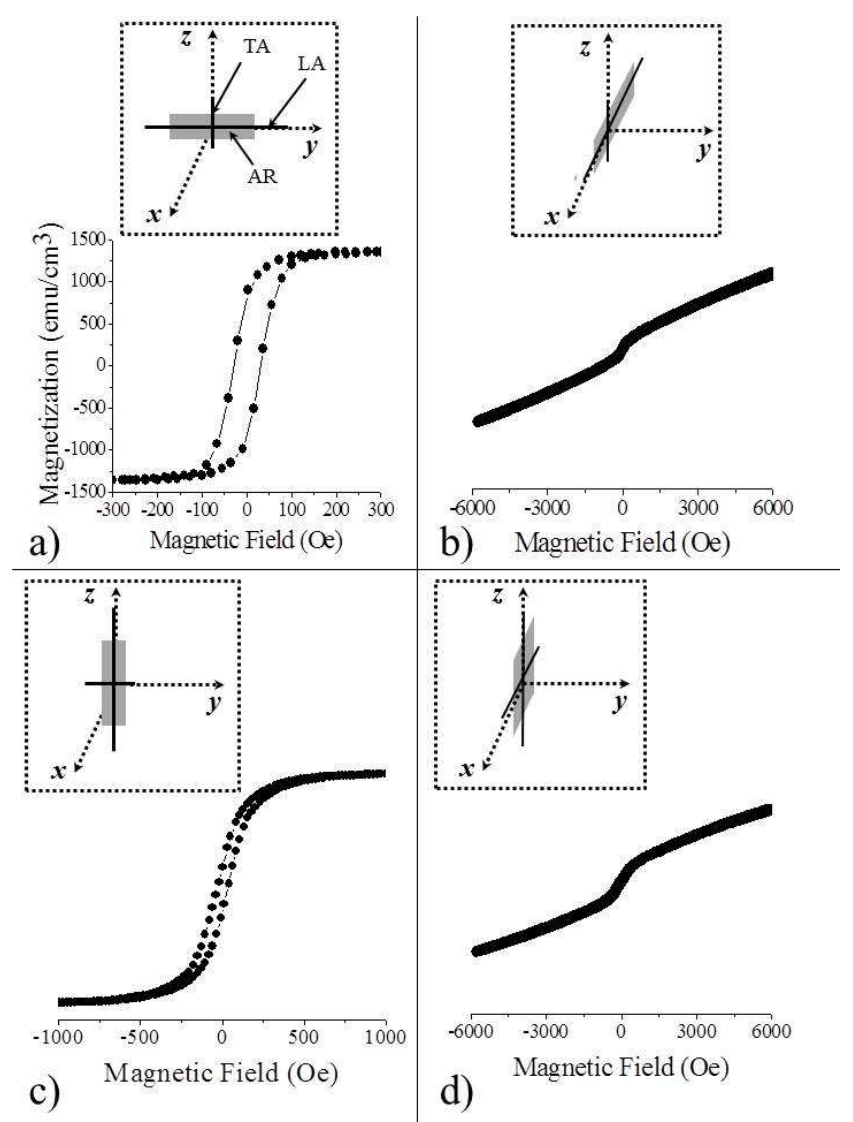

Figure 1. Hysteresis loop in configurations a) P10, b) P190, c) P20 and d) $P 290$. The box with dotted line represents the region of magnetic field; the field is generated by the electromagnet poles.

Sample temperatures were estimated by monitoring the temperature dependence of chosen the physical properties (retentivity $\mathrm{M}_{R}$, saturation magnetization $\mathrm{M}_{\mathrm{S}}$ and coercive field $\mathrm{H}_{\mathrm{C}}$ ) and changes of this properties according the orientation of ribbon plane to field. In Fig. 2, a typical hysteresis loop is shown. $\mathrm{M} / \mathrm{M}_{\mathrm{S}}$ as a function of field present: $M_{R},\left(H_{D C}=0\right), M_{S}$ saturation state (the magnetization vector is only in one direction) and $\mathrm{H}_{\mathrm{C}}($ where $\mathrm{M}=0)$. Three important magnetization processes we can distinguish: the domain bulging, the domain walls displacement and the spin rotation. This hysteresis loop corresponds to amorphous ribbon in as cast AC (sample with no thermal treatment) state at orientation P10, by increasing the magnetic field; the transverse domains respond quickly to fields above $200 \mathrm{Oe}$, the saturation state is achieved. This is because the ribbon 
contains $1 \% \mathrm{Cu}$ and thus a minimal energy is needed to achieve monodomain state. A comparison was made between orientation P10 and orientation P20 for the as cast ribbon, both hysteresis loops are shown in Fig. 3. We note that in the case of orientation P20, the monodomain state is reached until the fields above $1000 \mathrm{Oe}$, this is explained in terms of the amount of $\mathrm{Fe}(73.5 \%)$; it means that the system needs more power to move more moments, these are located along the longitudinal axis of ribbon, i.e. more energy is needed to overcome the spin-orbit coupling. Thus, depending on the geometric arrangement of the ribbon, we have a characteristic response. At P10, $M_{R}=815 \mathrm{emu} / \mathrm{cm}^{3}, M_{S}=1353 \mathrm{emu} / \mathrm{cm}^{3}$; at P20, $M_{R}=$ $216 \mathrm{emu} / \mathrm{cm}^{3}, \mathrm{M}_{\mathrm{S}}=1300 \mathrm{emu} / \mathrm{cm}^{3}$. At P10 the most notable difference is in the retentivity, it could be explained as follows, to change the field direction, this field requires moving a large number of moments (which are along the longitudinal axis) to bring it to a state of lower energy $\left(\mathrm{H}_{\mathrm{DC}}=0\right)$; not for orientation $\mathrm{P} 20$, since in this case is reduced the number of moments that are in the transverse axis.

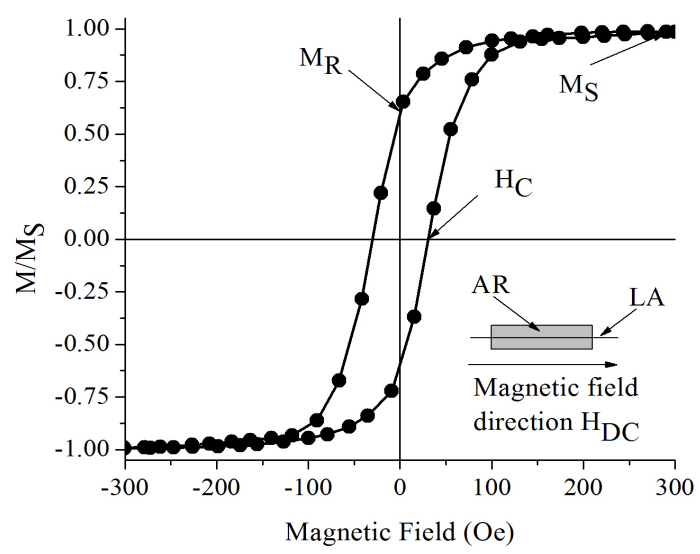

Figure 2. Hysteresis loop in orientation P10 for as cast state.

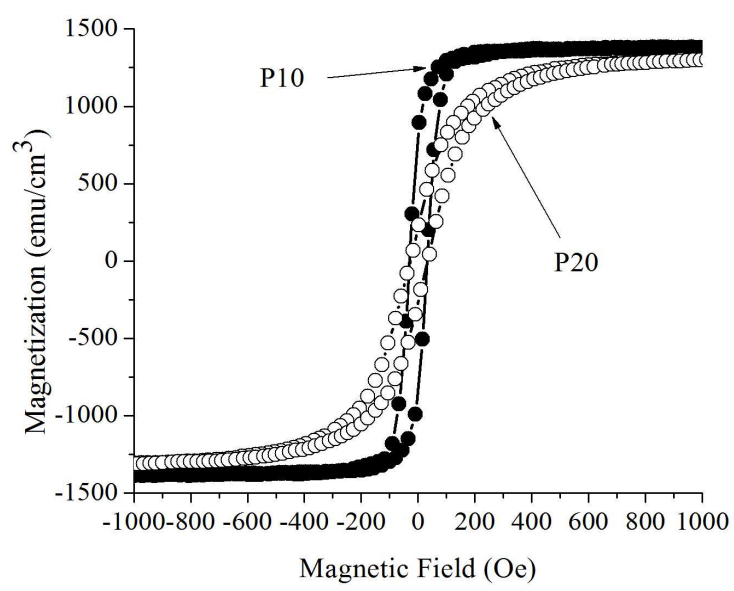

Figure 3. Comparison between $P 10$ and P20 in as cast state.

In a simple physical model, if $\mathrm{M}_{\mathrm{S}}$ in any one domain makes an angle $\theta$ with the positive field direction, the magnetization is $M_{S} \cos \theta$, the retentivity $M_{R}$ in whole ribbon is given by

$$
M_{R}=\int_{0}^{\pi / 2} M_{S} \cos \theta \sin \theta d \theta=M_{S} / 2
$$

From (2), we define $M_{R} / M_{S}$ the retentivity ratio [8]. According with this model, we might qualitatively estimate the energy involve in the dynamic of domain walls. The $M_{R} / M_{S}$ ratio at P10: $0.705,0.704,0.66$ and 0.55 for $10,40,60$ and $120 \mathrm{~min}$, respectively; at P20: 0.161, 0.141, 0.119 and 0.153 for $10,40,60$ and $120 \mathrm{~min}$, respectively. These experimental results together with the theoretical model are in correspondence with the physical explanation given above. As the direction of easy axis of the ribbon is the direction of spontaneous magnetization in the demagnetized state, we can correlate the retentivity ratio with the anisotropy energy. In extreme cases, if the ratio is close to 1 , indicates that the energy required to moving the vector of magnetization from its easy axis would be near to zero, if the ratio is near zero, the energy required to move the vector would be almost infinite.

From this way with the retentivity ratio is possible to determine the evolution of anisotropy depending not only the heat treatment time, but also on the orientation of amorphous alloy relative to the field. According to Cullity's model [9], with a very good approximation, to one-dimensional materials, the anisotropy calculation $\mathrm{K}_{1}$ is performed by using (3),

$$
H=2 \frac{K_{1}}{M_{S}}
$$

$\mathrm{H}$ is the magnetic field where the material is in saturation state. Anisotropy values $\mathrm{K}_{1}$ in orientation P10 are: $2.5 \times 10^{5}$, $19 \times 10^{5}, 1.81 \times 10^{5}$ and $1.86 \times 10^{5} \mathrm{erg} / \mathrm{cm}^{3}$ for $10,40,60 \mathrm{y} 120$ min, respectively; in orientation P20 these values are: $5.6 \times 10^{5}$, $4.42 \times 10^{5}, 4.46 \times 10^{5}$ and $4.89 \times 10^{5} \mathrm{erg} / \mathrm{cm}^{3}$ for $10,40,60 \mathrm{y} 120$ min, respectively. In Fig. 4, the hysteresis loop for the ribbon with no thermal treatment at positive fields is shown. The induced anisotropy by the thermal treatment in the amorphous ribbon was evaluated qualitatively from the area formed by curve of hysteresis loop and the horizontal line located where the magnetization saturation is achieved.

The induced anisotropy is an important design parameter. In many works has already showed that step-induced anisotropy can be used in the development of magnetoresistive sensors based on Hall effect and spin-dependent tunneling [10,11]. We use the anisotropy energy $\mathrm{K}_{1}$ as a design parameter. In P10, a shaded area is indicated and it is proportional to the anisotropy energy. In the inset of the figure, a shaded area is indicated for the case P20. This behavior is very important due to it sets the initial conditions of the anisotropy energy and we can see the change with the annealing time. Most pronounced change in properties of the amorphous ribbon and the energy involved in the dynamics of the magnetic moments is where the orientation of the magnetization vector is out plane $\{\mathrm{P} 190$, $\mathrm{P} 290$ \}. Even though the saturation is not achieved, we use the Eq. 3 with $\mathrm{M}_{\mathrm{S}}=6000$ Oe.

The Fig. 5 shows a shaded area for orientation P190, this area is proportional to the stored energy by the magnetic moments when they are oriented to the applied field direction. The inset shows the behavior of the ribbon in orientation P290. Clearly we note that the shaded area has changed, so $\mathrm{K}_{1}$ is a very sensitive parameter of position. In both curves is not possible to 
determine the retentivity and the saturation magnetization, we can explain it as follow, the applied field is normal to ribbon plane, so the field do not distinguish the magnetic structure which is formed by domains and domain walls.

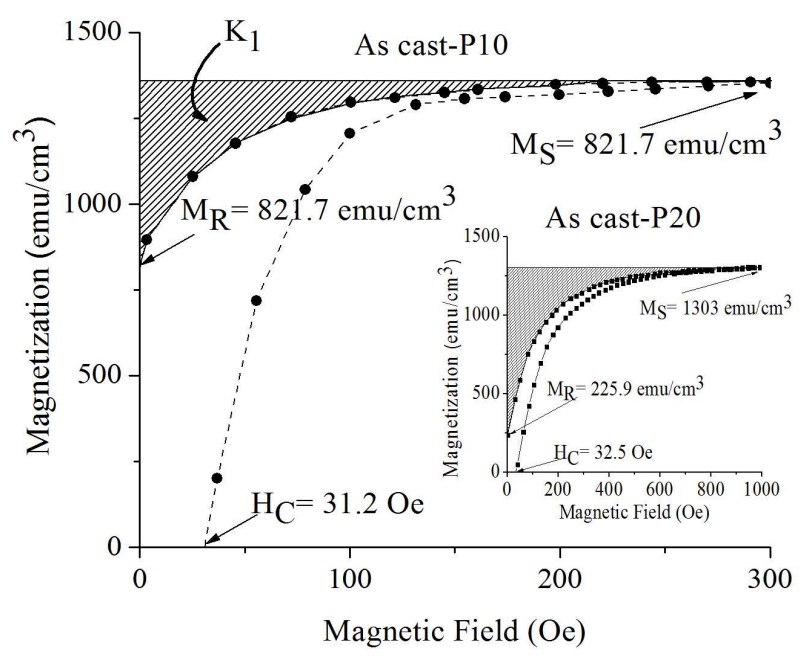

Figure 4. Magnetization as a function of field for as cast at P10 and P20 (in the inset), $K_{I}$ is related to induced anisotropy.

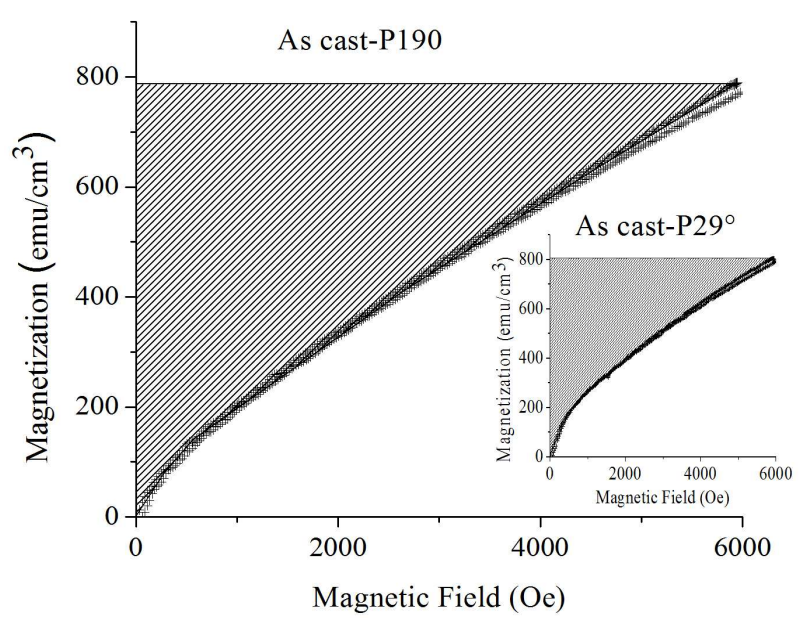

Figure 5. Anisotropy energy in orientation P190 and orientation P290 (inset).The shaded area is proportional to induced anisotropy due to the thermal treatment.

The magnetic properties in P190 and P290 are shown in Table 1. The column 2 and the column 3 corresponds to P190 (with a maximum value $\mathrm{M}_{\mathrm{S}}=798 \mathrm{emu} / \mathrm{cm}^{3}, \mathrm{~K}_{1}=2.37 \times 10^{6}$ $\left.\mathrm{erg} / \mathrm{cm}^{3}\right)$, the column 4 and the column 5 corresponds to $\mathrm{P} 290$ (with a maximum value $\mathrm{M}_{\mathrm{S}}=802 \mathrm{emu} / \mathrm{cm}^{3}, \mathrm{~K}_{1}=2.41 \times 10^{6}$ $\left.\mathrm{erg} / \mathrm{cm}^{3}\right)$.

Table 1. The magnetic properties in P190 and P290. As cast (AC).

\begin{tabular}{|c|c|c|c|c|}
\hline $\begin{array}{l}\text { Time } \\
\text { (min) }\end{array}$ & $\begin{array}{l}\text { Magnetization } \\
\text { Saturation, } \\
M_{S}\left(\mathbf{e m u} / \mathbf{c m}^{3}\right)\end{array}$ & $\begin{array}{l}\text { Anisotropy } \\
\text { Energy, } K_{1} \\
\left(\times 10^{6}\right. \\
\left.\text { erg/cm }{ }^{3}\right)\end{array}$ & $\begin{array}{l}\text { Magnetization } \\
\text { Saturation, } \\
M_{S}\left(\mathbf{e m u} / \mathbf{c m}^{3}\right)\end{array}$ & $\begin{array}{l}\text { Anisotropy } \\
\text { Energy, } K_{1} \\
\left(\times 10^{6}\right. \\
\left.\mathrm{erg}^{6} \mathrm{~cm}^{3}\right)\end{array}$ \\
\hline $\mathrm{AC}$ & 798 & 2.37 & 802 & 2.41 \\
\hline 10 & 714 & 2.14 & 670 & 2.0 \\
\hline 40 & 515 & 1.55 & 522 & 1.57 \\
\hline 60 & 568 & 1.70 & 550 & 1.65 \\
\hline 120 & 455 & 1.40 & 374 & 0.6 \\
\hline
\end{tabular}

The effect of the annealing time over the magnetic properties in the alloy is shown in Fig. 6. Fig. 6a shows the dependencies of $M_{R} / M_{S}$ and $M_{R}$ on annealing time at P10. It can be seen that $M_{R} / M_{S}$ changes very regular at annealing. $M_{R}$ increases with increasing the time of annealing treatment until a maximum value of $870 \mathrm{emu} / \mathrm{cm}^{3}$ (for as cast), and then its consequent decrease until a minimum value of $516 \mathrm{emu} / \mathrm{cm}^{3}$ (for $120 \mathrm{~min}$ ). A similar behavior we found in the retentivity ratio. $M_{R} / M_{S}$ increases with increasing the annealing time until a maximum value of 0.705 (for $10 \mathrm{~min}$ ), and then its decrease until a minimum value of 0.55 (120 min).

Fig. $6 \mathrm{~b}$, shows the dependencies of $\mathrm{H}_{\mathrm{C}}$ and $\mathrm{M}_{\mathrm{S}}$ on annealing time. The $\mathrm{H}_{\mathrm{C}}$ initially decreases until a minimum 31 Oe, corresponding to amorphous ribbon with $40 \mathrm{~min}$ of thermal treatment, after 40 min $\mathrm{H}_{\mathrm{C}}$ increases slowly to 60 min with 34 Oe. Then $\mathrm{H}_{\mathrm{C}}$ decreases slowly to 120 min with 29 Oe. This behavior is due to magnetic softening originated by structural relaxation. It can be explained as the nucleation of a nanocrystal precursor matrix. This nanocrystals has a large anisotropy that the amorphous phases and are poorly couple to it $[12,13]$, indicating that they are acting as effective pinning centers for the propagating domains $[14,15] . \mathrm{M}_{\mathrm{S}}$ decreases slowly until a minimum value of $455 \mathrm{emu} / \mathrm{cm}^{3}$. This behavior has been reported in other works [16], and it has been explained as a softening and hardening state, respectively. In order to guarantee the magnetic softness of the amorphous ribbon with ultrafine FeMo structure, it is important to inhibit the formation of Fe-borides which is managed by the molybdenum addition in combination with the boron content [17].
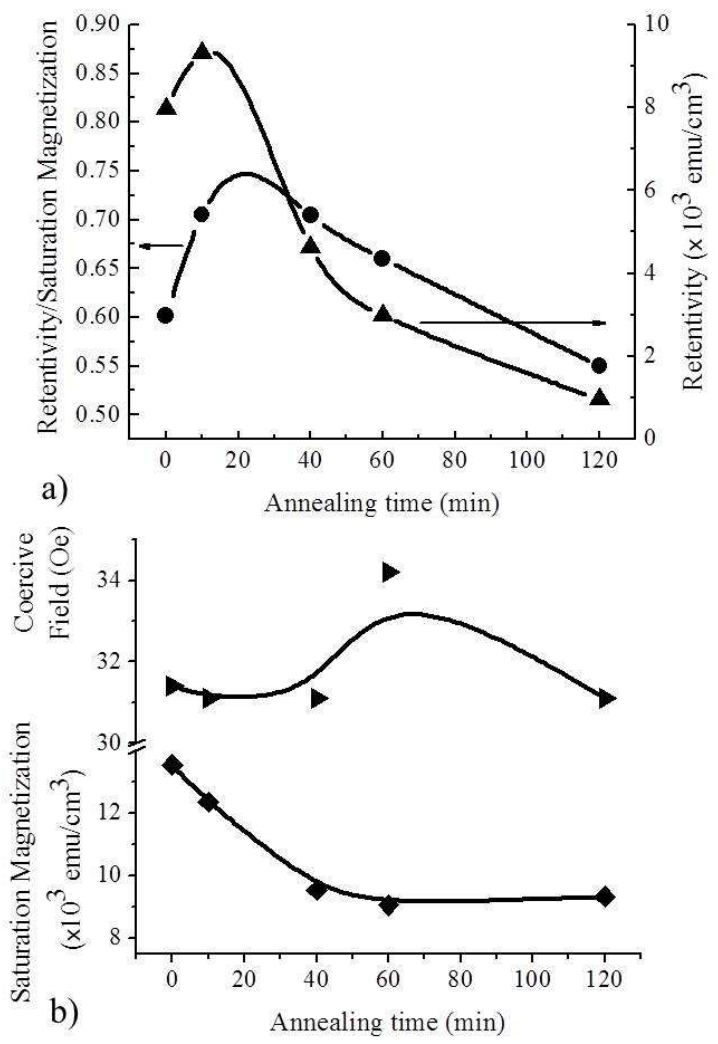

Figure 6. a) Retentivity, retentivity ratio, b) coercive field and saturation magnetization as a function of annealing time. 
The response of the ribbon for orientation P190 is shown in Fig. 7. This behavior is very interesting, since the magnetization increases linearly, the rate of change of the magnetization is defined as $m=\Delta \mathrm{M} / \Delta \mathrm{H}$, we find the values of 0.122 (700/5776.9), 0.107 (563/5254), 0.069 (388/5582), $0.063(346 / 5507)$ and $0.075(406 / 5411)$ emu / Oe $\mathrm{cm}^{3}$ corresponding to as cast, 10, 40, 60 and $120 \mathrm{~min}$, respectively. For the as cast, we find the largest range of magnetic field detection. The minimum is for $10 \mathrm{~min}$. According the magnetization change with the field, the maximum is for the as cast and the minimum for $60 \mathrm{~min}$. In orientation P290, the values of the rate of change of magnetization are 0.11 (568/5157), 0.84 (437/5183), 0.089 (458/5137), 0.08 (334/4173) and $0.11(322 / 2913)$ emu / Oe $\mathrm{cm}^{3}$ corresponding to as cast, 10, 40,60 and $180 \mathrm{~min}$, respectively. For $10 \mathrm{~min}$, we found the largest range of magnetic field detection. The minimum is for $180 \mathrm{~min}$. According the magnetization change with the field, the maximum is for the as cast and the minimum for $180 \mathrm{~min}$.

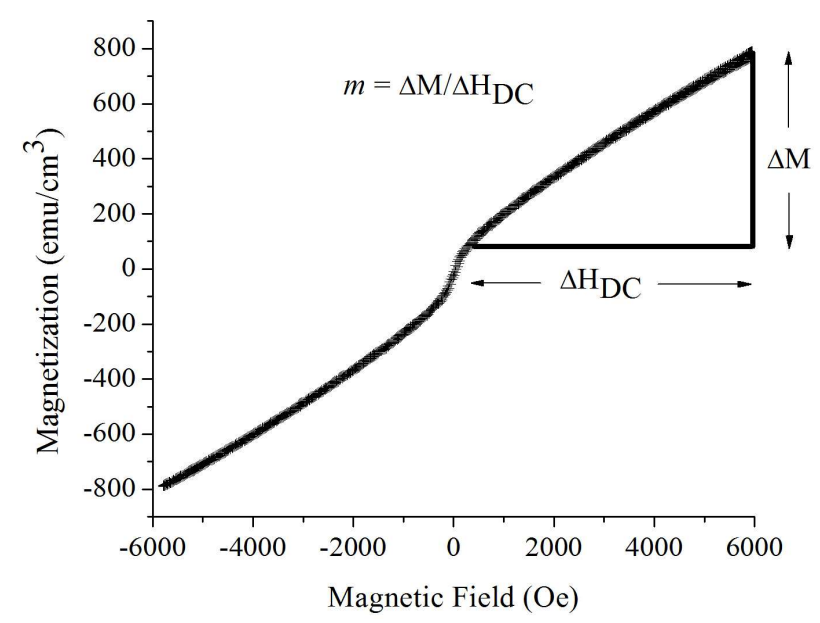

Figure 7. Rate of change of magnetization (slope of linear region in the hysteresis loop.

The variation of sensibility as a function of annealing time is shown in table 2. This is the major parameter to determine the response of the amorphous ribbon in front of DC magnetic field. There is a direct relationship between rate of change of magnetization and the saturation magnetization. A material with a high value of $\mathrm{M}_{\mathrm{S}}$ is a very good candidate to design magnetic field sensors. The column 2 corresponds to orientation P190 and the column 3 corresponds to orientation P290. In both cases the maximum value is for the material with no thermal treatment (as cast) and the minimum value is for $60 \mathrm{~min}$.

Table 2. The magnetization ratio to $P 190$ and $P 290$.

\begin{tabular}{|c|c|c|}
\hline & $\Delta \mathrm{M} / \Delta \mathrm{H}\left(\mathrm{emu} / \mathrm{Oe} \mathrm{cm}^{3}\right)$ & $\Delta \mathrm{M} / \Delta \mathrm{H}\left(\mathrm{emu} / \mathrm{Oe} \mathrm{cm}^{3}\right)$ \\
\hline As Cast & 0.122 & 0.110 \\
\hline $10 \mathrm{~min}$ & 0.107 & 0.084 \\
\hline $40 \mathrm{~min}$ & 0.069 & 0.089 \\
\hline $60 \mathrm{~min}$ & 0.063 & 0.08 \\
\hline $120 \mathrm{~min}$ & 0.075 & ----- \\
\hline $180 \mathrm{~min}$ & ----- & 0.11 \\
\hline
\end{tabular}

The evolution of magnetization as a function of heat treatment time to P190 is shown in Fig. 8. In all curves, we can see a clear change in the slope and consequently in the sensibility. This change gives information about the response of the amorphous ribbon with the temperature. An important requirement is that the shape of the hysteresis loop can be varied according to the necessities of technological applications.

An atomic pair ordering due to a thermal treatment induces an easy direction parallel to the applied field. It has been widely discussed elsewhere that this behavior is due to the evolution of the amorphous and nanocrystalline phases, the selected temperature is below the crystallization (500 and $630^{\circ} \mathrm{C}$ ) [18]. All these factors affect to dynamic of the magnetic moments. The change of amorphous and nanocrystalline phases determines the hysteresis form. Hysteresis loop of the treatment samples are quite different when comparing to as cast. The different results obtained through the thermal treatment method show that the field induced anisotropies behave in a very different way with respect to the induced anisotropies by orientation P10 and orientation P20.

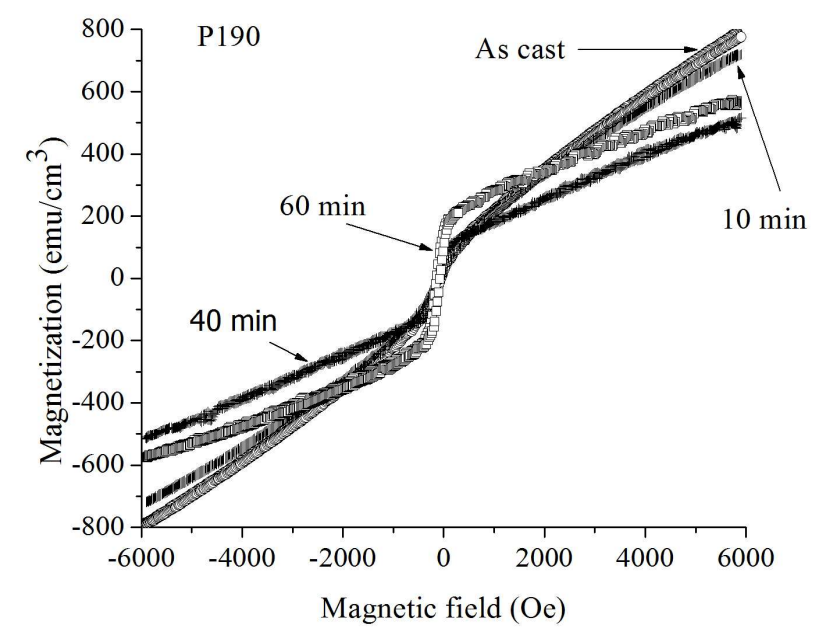

Figure 8. Variation of slope in the hysteresis loop with different annealing times.

For future works, we can determine the magnetic properties and the linear response of the magnetization at temperatures above the first and second nanocrystallization phase. Joule treatment is feasible, the purpose is to determine the stability of the material against an alternating current and thereby determine the critical point at which the magnetic properties such as anisotropy, saturation magnetization, and retentivity affected. In the design of any sensor is vital to know the thermal stability. This amorphous ribbon is wonderful, since according to their various responses can also be used as a temperature sensor.

The knowledge of the change of the anisotropy of the amorphous ribbon, not only with the sample orientation with respect to the field, but with the heat treatment time, a comprehensive methodology is set for declare that this material is a serious candidate for industrial applications. 


\section{Conclusions}

The amorphous ribbon was studied in four geometric arrangements: P10, P190, P20 and P290. Changes of the retentivity, saturation magnetization, anisotropy energy, coercive field and $\Delta \mathrm{M} / \Delta \mathrm{H}$ in $\mathrm{Fe}_{73.5} \mathrm{~B}_{9} \mathrm{Si}_{13.5} \mathrm{Mo}_{3} \mathrm{Cu}_{1}$ amorphous alloy have been reported. The response of $\mathrm{M}_{R}, \mathrm{M}_{S}, \mathrm{M}_{\mathrm{R}} / \mathrm{M}_{\mathrm{S}}$ and $\mathrm{K}_{1}$ was analyzed, not only in terms of the geometrical arrangement, but also in terms of time of heat treatment. The best values are presented for orientation P10. In the case of the orientation $\mathrm{P} 10$, at as cast ribbon, the values are: $\mathrm{M}_{\mathrm{S}}=1353$ $\mathrm{emu} / \mathrm{cm}^{3}, \mathrm{M}_{\mathrm{R}}=815 \mathrm{emu} / \mathrm{cm}^{3}$ and $\mathrm{M}_{\mathrm{R}} / \mathrm{M}_{\mathrm{S}}=0.6016$; at P20: $\mathrm{M}_{\mathrm{S}}$ $=1300 \mathrm{emu} / \mathrm{cm}^{3} \mathrm{M}_{\mathrm{R}}=216 \mathrm{emu} / \mathrm{cm}^{3}$, and $\mathrm{M}_{\mathrm{R}} / \mathrm{M}_{\mathrm{S}}=0.166$, respectively. However, for orientation P20 a larger anisotropy value is achieved $K_{1}=650,000 \mathrm{erg} / \mathrm{cm}^{3}$ than to the case of orientation P10 $\left(\mathrm{K}_{1}=270.600 \mathrm{erg} / \mathrm{cm}^{3}\right)$. This difference is generated because at P10 the magnetization vector is in the ribbon plane, and the dynamic vector is regulated by the magnetic structure (domains and domain walls). In P20, the dynamic magnetization vector is outside the ribbon plane, so the domain dynamic is not regulated by the magnetic structure, in this case exchange interaction takes less importance.

The highest values of anisotropy are presented for $\mathrm{P} 190$ and P290 arrangements, these values for as cast are: $K_{1}=$ $2,365,100 \mathrm{erg} / \mathrm{cm}^{3}$ and $\mathrm{K}_{1}=2,405,520 \mathrm{erg} / \mathrm{cm}^{3}$, respectively. Regarding the rate of change $\Delta \mathrm{M} / \Delta \mathrm{H}$ (defined only for the study of the amorphous ribbon in P190 and P290), we have the following values. At P190: 0.122, 0.107, 0.069, 0.063 and $0.075 \mathrm{emu} / \mathrm{Oe} \mathrm{cm}^{3}$ corresponding to as cast, 10, 40, 60 and 120 min, respectively; At P290: 0.11, 0.084, 0.089, 0.08 and $0.11 \mathrm{emu} / \mathrm{Oe} \mathrm{cm} \mathrm{cm}^{3}$ corresponding to as cast, $10,40,60$ and 180 min, respectively. The best linear increase is for the ribbon without heat treatment.

According to our results, the amorphous alloy is a strong candidate as a vector field sensor in three directions: longitudinal, transverse and normal to the plane (saturation magnetization, retentivity and external magnetic field). In the subsequent, detailed study considering small steps of heat treatment time, in order to determine the critical point where the magnetic properties begin to change, so we can control the thermal stability of the amorphous ribbon.

\section{References}

[1] W. H. Meiklejohn and C. P. Bean, "New Magnetic Anisotropy," Physical Review, vol. 105, N. 3, pp. 904-913, February 1957.

[2] Andrei V. Palii, Boris S. Tsukerblat, Eugenio Coronado, Juan M. Clemente-Juan, Juan J. Borrás-Almenar, "Orbitally dependent kinetic Exchange in cobalt (II) pairs:origin of the magnetic anisotropy," Polyhedron, vol. 22, pp. 2537-2544, February 2003.

[3] Giselher Herzer, "Nanocrystalline soft magnetic materials," Journal of Magnetism and Magnetic Materials, vol. 157/158, pp. 133-136, 1996.

[4] Tadeusz Kulik, Antonio Hernando,"Magnetic properties of
$\mathrm{Fe}_{76.5-x} \mathrm{Cu}_{1} \mathrm{Nb}_{x} \mathrm{Si}_{13.5} \mathrm{~B}_{9}$ alloys nanocrystallized from amorphous state," Journal of Magnetism and Magnetic Materials, vol. 160, pp. 269-270, 1996.

[5] V. Franco, C. F. Conde, A. Conde, "Changes in magnetic anisotropy distribution during structural evolution of $\mathrm{Fe}_{76} \mathrm{Si}_{10.5} \mathrm{~B}_{9.5} \mathrm{Cu}_{1} \mathrm{Nb}_{3}$," Journal of Magnetism and Magnetic Materials, vol. 185, pp. 353-359, 1998.

[6] Simon Foner, "Versatile and Sensitive Vibrating-Sample Magnetometer," The Review of Scientific Instruments, vol. 30, pp. 548-557, July 1959.

[7] David Jiles, Introduction to Magnetism and Magnetic Materials, 1st ed., Chapman and Hall, 1991, pp. 49-52.

[8] Shoshin Chikazumi, Physics of Magnetism, $1^{\text {st }}$ ed., John Wiley and Sons, 1964, pp. 554.

[9] B. D. Cullity, Introduction to Magnetic Materials, Addison-Wesley Publishing Company, Inc., 1972, pp. 225-229.

[10] R. S. Popovic, J. A. Flanagan, P. A. Besse, "The future of magnetic sensors," Sensors and Actuators A, vol. 56, pp. 39-55, 1996.

[11] G. Herzer, M. Vázquez, M. Knobel, A. Zhukov, T. Reininger, H. A. Davies, R. Grössinger, J. L. Sánchez Ll, "Round table discussion: Present and future applications of nanocrystalline magnetic materials," Journal of Magnetism and Magnetic Materials, vol. 294, pp. 252-266, 2005.

[12] C. Miguel, A. Zhukov, J. J. del Val. J. Gonzáles, "Coercivity and induced magnetic anisotropy by stress and/or field annealing in $\mathrm{Fe}$ - and Co- based (Finemet-type) amorphous alloys," Journal of Magnetism and Magnetic Materials, vol. 294, pp. 245-251, 2005.

[13] N. Murillo, J. González, "Effect of the annealing conditions and grain size on the soft magnetic character of $\mathrm{FeCu}(\mathrm{Nb} / \mathrm{Ta}) \mathrm{SiB}$ nanocrystalline alloys," Journal of Magnetism and Magnetic Materials, vol. 218, pp. 53-59, March 2000.

[14] R. Schäfer, S. Roth, C. Stiller J. Eckert, U. Klement and L. Schultz, " Domain Studies on Mechanically Alloyed $\mathrm{Fe}-\mathrm{Zr}$-B-Cu- Nanocrystalline Powder," IEEE Transactions on Magnetics, vol. 32, No. 5, pp. 4383-4385, 1996.

[15] R. Valenzuela and J. T. S. Irvine, "Domain Wall dynamics and short-range order in ferromagnetic amorphous ribbons," Journal of Non-Crystalline Solids, vol. 156-158, pp. 315-318, 1993.

[16] Arturo Mendoza Castrejón, Herlinda Montiel Sánchez, Guillermo Alvarez Lucio, Rafael Zamorano Ulloa, "Nanocrystallization in $\mathrm{Fe}_{73.5} \mathrm{Si}_{13.5} \mathrm{~B}_{9} \mathrm{Mo}_{3} \mathrm{Cu}_{1}$ amorphous ribbon and its magnetic properties," Materials Science Forum, vol. 691, pp. 77-82, 2011.

[17] P. Kwapuliński, A. Chrobak, G. Haneczok, Z. Stokłlosa, J. Rasek, "Structural relaxation and magnetic properties of $\mathrm{Fe}_{86-x} \mathrm{Nb}_{x} \mathrm{~B}_{14}$ amorphous alloys," Journal of Magnetism and Magnetic Materials, vol. 304, pp. e654-e656, March 2006.

[18] E. Illeková, D. Janičkovič, M. Miglierini, I. Škorvánek, P. Švec, "Influence of $\mathrm{Fe} / \mathrm{B}$ ratio on thermodynamic properties of amorphous Fe-Mo-Cu-B," Journal of Magnetism and Magnetic Materials, vol. 304, pp. e636-e638, March 2006. 